\title{
Le modèle organisationnel de la Bibliothèque de l'Université du Québec à Chicoutimi
}

\section{The Organisational Model Operating in the Library at the Université du Québec à Chicoutimi El modelo organizacional de la Biblioteca de la Universidad de Quebec en Chicoutimi}

\section{Gilles Caron}

Volume 40, numéro 3, juillet-septembre 1994

URI : https://id.erudit.org/iderudit/1033471ar

DOI : https://doi.org/10.7202/1033471ar

Aller au sommaire du numéro

Éditeur(s)

Association pour l'avancement des sciences et des techniques de la documentation (ASTED)

ISSN

0315-2340 (imprimé)

2291-8949 (numérique)

Découvrir la revue

Citer cet article

Caron, G. (1994). Le modèle organisationnel de la Bibliothèque de l'Université du Québec à Chicoutimi. Documentation et bibliothèques, 40(3), 121-132.

https://doi.org/10.7202/1033471ar
Résumé de l'article

Qualité totale, organisation matricielle, gestion par équipes, ré-ingénierie : les sciences de la gestion nous ont habitués à une révision périodique du vocabulaire associé à la gestion de nos organisations. Malheureusement, peu d'exemples concrets d'implantation réussie de tels systèmes nous sont présentés et, surtout, encore moins d'organisations sont en mesure de proposer une approche conceptuelle rationnelle de la mise en place de tels changements. Depuis plus de douze ans maintenant, la Bibliothèque de l'Université du Québec à Chicoutimi (UQAC) vit un modèle organisationnel original dont on retrouve peu d'exemples en Amérique du nord. Ce modèle privilégie la satisfaction des besoins d'information de ses clients comme objectif principal de l'établissement.
Tous droits réservés (c) Association pour l'avancement des sciences et des techniques de la documentation (ASTED), 1994
Ce document est protégé par la loi sur le droit d'auteur. L’utilisation des services d’Érudit (y compris la reproduction) est assujettie à sa politique d'utilisation que vous pouvez consulter en ligne. 


\title{
Le modèle organisationnel de la Bibliothèque de l'Université du Québec à Chicoutimi
}

\author{
Gilles Caron \\ Directeur de la Bibliothèque Paul-Émile-Boulet \\ Université du Québec à Chicoutimi
}

Qualité totale, organisation matricielle, gestion par équipes, ré-ingénierie: les sciences de la gestion nous ont habitués à une révision périodique du vocabulaire associé à la gestion de nos organisations. Malheureusement, peu d'exemples concrets d'implantation réussie de tels systèmes nous sont présentés et, surtout, encore moins d'organisations sont en mesure de proposer une approche conceptuelle rationnelle de la mise en place de tels changements. Depuis plus de douze ans maintenant, la Bibliothèque de l'Université du Québec à Chicoutimi (UQAC) vit un modèle organisationnel original dont on retrouve peu d'exemples en Amérique du nord. Ce modèle privilégie la satisfaction des besoins d'information de ses clients comme objectif principal de l'établissement.

\section{The Organisational Model Operating in the Library at the Université du Québec à Chicoutimi}

Total quality, organisational matrices, team management, and reengineering are terms that have cropped up in the vocabulary of management sciences. Unfortunately, there are few documentated examples, of the successful implementation of such systems and few organisations have been able to propose the conceptual framework necessary for such changes. For the last twelve years, the Library at the Université du Québec à Chicoutimi has experienced a unique model rarely seen in North American institutions. The principal objective of this model lies in the satisfaction of the information needs of its clients.

\section{El modelo organizacional de la Biblioteca de la Universidad de Quebec en Chicoutimi}

Las ciencias de la gestión nos han habituado a una revisión periódica del vocabulario asociado a la gestión de nuestras organizaciones: cualidad total, organización dirigente, gestión por equipos, re-ingeniería. Desgraciadamente, pocos ejemplos concretos de aplicación que han tenido éxito de tales sistemas nos son presentados $y$, sobre todo, menos aún las organizaciones pueden proponer un enfoque conceptual racional de la implantación de estos cambios. Desde más de doce meses, la Biblioteca de la Universidad de Quebec en Chicoutimi (UQAC) cuenta con un modelo organizacional original del cual encontramos pocos ejemplos en América del Norte. Este modelo privilegiala satisfacción de las necesidades de los usuarios como objetivo principal del establecimiento.
La bibliothéconomie est confrontée à un problème profond d'identité à la suite de l'émergence de la société post-industrielle caractérisée, entre autres choses, par la prolifération des informations disponibles et l'expansion rapide des moyens de communications.

Les bibliothèques et les bibliothécaires se voient défiés sur leur propre terrain par des organismes et des individus qui se présentent comme fournisseurs ou diffuseurs d'informations sur la base du fait qu'ils ont accès parterminal à une base de données quelconque, qu'ils maîtrisent l'infrastructure de communication ou plus simplement encore qu'ils produisent l'équipement technique nécessaire. De la société IBM aux producteurs de contenus en passant par les «transporteurs" de tout acabit, tous se targuent d'appartenir désormais au secteur de l'information. La compétition dans ce secteur s'annonce féroce et à la mesure des enjeux financiers que l'on prévoit énormes

Confrontées à une telle concurrence, de plus en plus de personnes au sein de notre profession envisagent la situation apocalyptique où, ayant été poussés dans leurs derniers retranchements, les bibliothécaires deviendront les gardiens de quantité considérable de documents de plus en plus dépassés, isolés dans une sorte de "no man's land» culturel entre l'archiviste et ces nouveaux fournisseurs d'informations qui, éventuellemient, récupéreront à leur profit la considération du public et de la société. Cette angoisse est présente en permanence dans la littérature bibliothéconomique contemporaine, entraînant des réactions diverses au sein de la profession. Certains tentent de s'approprier «tout ce qui bouge» dans une course frénétique pour s'accaparer toutes les nouveautés sinon les modes technologiques qui surgissent quotidiennement, alors que d'autres, par contre, se réfugient derrière l'imprimé, sinon le livre, perçu comme immortel, donc invulnérable.

Ce débat est présent en permanence au sein même de nos bibliothèques. Bien plus, il est exacerbé par le modèle organisationnel en place dans la plupart de nos bibliothèques académiques. Le modèle organisationnel traditionnel est en lui- 
même l'illustration de cette dichotomie. Les services au public se perçoivent comme les défenseurs de l'accès à l'information alors que les services dits techniques demeurent les bastions du document considéré comme la raison d'être de la bibliothèque. Notre ambivalence est la conséquence logique sinon nécessaire du contexte structurel imposé par l'organisation bibliothèque à la profession. De fait, il demeurera toujours très difficile de résoudre cette dichotomie sans une réflexion d'importance sur la bibliothèque comme organisation et plus profondément sur les finalités de la profession qui est la nôtre au sein de ce type d'organisation.

Plusieurs auteurs au cours des dix dernières années ont publié des textes portant sur les défis confrontant notre profession et les bibliothèques. De fait, le nombre d'écrits portant sur «la bibliothèque de l'an 199?... et plus» est considérable. Certains ont proposé des modèles nouveaux d'organisation, de nouvelles façons d'être et de faire, d'utiliser le personnel, etc. De Martell à Jennings ${ }^{2}$, en passant par plusieurs autres ${ }^{3}$, chacun $y$ est allé de sa vision sur le sujet et de sa recette qui permettrait de négocier le virage susceptible de réconcilier les bibliothécaires et les bibliothèques avec leur avenir en maintenant leur position comme principal fournisseur d'information à leurs usagers respectifs. Malheureusement, comme l'ont reconnu certains auteurs ${ }^{4}$, la «litterature on innovation is a call for its exercise and/or a list of possible areas of need or application» plus que l'illustration de réalisations concrètes.

A la Bibliothèque de l'Université du Québec à Chicoutimi, nous avons proposé notre solution et l'avons appliquée. Depuis plus de douze ans, cette bibliothèque universitaire fonctionne sur la base d'une vision cohérente de ce que doit être sa finalité et sa raison d'être. Nous fondant sur cette vision, nous avons développé des orientations et mis en place un modèle organisationnel qui, nous le croyons, a permis à la bibliothèque de se réconcilier avec elle-même et aux membres de notre personnel d'évoluer dans un contexte stimulant susceptible de leur permettre de livrer leur plein potentiel à la fois comme individu et comme travailleur intellectuel. C'est là l'objet du présent article.

\section{L'UQAC et sa bibliothèque}

L'Université du Québec à Chicoutimi (UQAC) a été fondée en 1969. Elle est l'un des 11 établissements de l'Université du Québec, un réseau universitaire couvrant tout le territoire du Québec.

Le campus principal de I'UQAC est situé à Chicoutimi, une ville de 65000 habitants située à environ 200 kilomètres au nord de la ville de Québec. L'UQAC rayonne sur un territoire très étendu (400 000 kilomètres carrés) comptant environ 400000 personnes, dont 300000 dans la périphérie immédiate de l'UQAC, le Saguenay-Lac-Saint-Jean.

L'UQAC reçoit présentement 7500 étudiants (équivalant à 4300 étudiants à temps complet) inscrits à tous les cycles universitaires (du baccalauréat au doctorat). Une de ses caractéristiques est la grande dispersion de sa programmation, laquelle compte pas moins de 105 programmes actifs couvrant un éventail de disciplines extrêmement étendu. En pratique, seul le droit et la médecine n'y sont pas directement représentés quoique, encore là, des programmes connexes (nursing et droit des affaires) existent dans ces deux secteurs. Cette dispersion n'est d'ailleurs pas sans causer des problèmes importants.

La Bibliothèque de I'UQAC compte 41,4 employés, dont 11,4 professionnels incluant les trois cadres de la direction, 13 bibliotechniciennes et 17 commis. Le fonds documentaire est d'environ 850000 unités dont plus de 350000 imprimés. On y dispense la panoplie habituelle des services disponibles dans la plupart des bibliothèques universitaires modernes, $y$ compris l'accès réseau en ligne à un catalogue de bibliothèque (OPAC), et ce, depuis 1975 (BADADUQ, suivi de SIGIRD). Une des caractéristiques de la Bibliothèque est la grande importance accordée à la formation/information documentaire des usagers. Le programme touchait en 1992-93 plus de $60 \%$ des nouveaux inscrits au premier cycle dont près de $50 \%$ dans le cadre d'activités créditées.

\section{Des choix stratégiques préalables}

En 1979, à l'occasion de l'élaboration du projet de construction d'une nou- velle bibliothèque, la direction de la Bibliothèque entreprit une réflexion en profondeur sur les orientations qui devaient lui permettre de concrétiser sa mission de support à l'enseignement, à la recherche et au développement de la collectivité régionale. Cet exercice de planification stratégique devaitl'amener à certains constats qu'il serait bon de rappeler ici puisqu'ils servent de trame de fonds aux orientations retenues.

Le constat d'ensemble se résume à deux points:

1) La Bibliothèque de I'UQAC, compte tenu des ressources dont dispose et disposera l'Institution, ne pourra jamais espérer être en mesure de constituer sur place le fonds documentaire lui permettant de soutenir la gamme de programmes d'enseignement et de recherche susceptibles d'être développés à Chicoutimi.

2) Par ailleurs, nos étudiants et chercheurs n'en évoluent pas moins dans un contexte éminemment compétitif où l'accès à une information de pointe de qualité constitue une nécessité incontournable.

1. Charles Martell, The Client-Centered Academic Library, Westport, Greenwood Press, 1983, 136p.

2. Lois Jennings, «Regrowing Staff: Managerial Priority for the Future of University Libraries), Public-Access Computer Systems Review, vol. 3, no. 3 (1992), 4-15.

3. Sur la nécessité de revoir le modèle organisationnel au sein des bibliothèques, voir, outre Martell déjà cité: Anna A. Altmann, "The Academic Library of Tomorrow: Who will do what?n, Canadian Library Journal, vol. 45, no. 3 (June 1988), 147-152; Helen A. Howard, «Improving Services through the Redesign of Organizational Structures》 in Nos ressources humaines: la clé d'un bon service/Personnel: Key to successful public service, Montréal, Corporation des bibliothécaires du Québec, $16^{\circ}$ congrès, (mars 1985), p. 11-18; Peggy Johnson, «Matrix Management: AnOrganizational Alternative for Libraries),,Journal of Academic Librarianship, vol. 16, no. 4 (1990), 222-229. Pour un exemple récent d'une expérience en ce sens, voir: Stephen Ross and John Buschman, «The Compleat Library Organization: A Case Study of the Academic Library), Library Administration \& Management, vol. 7 , no. 2 (Spring 1993), 79-88.

4. Judy Reynolds and Jo Bell Whitlatch, «Academic Library Services: The Literature of Innovation», College \&ResearchLibraries,(September 1985), 409. 
Sans accès aù même bassin d'information que leurs confrères des autres établissements universitaires, il est illusoire de penser que nos professeurs et chercheurs puissent développer à Chicoutimi des programmes d'études avancées et de recherche un tant soit peu remarquables.

Compte tenu donc de l'importance de l'information dans la compétitivité de nos clients, il devenait inimaginable de penser réaliser la mission de l'UQAC sans disposer sur place, sinon des documents, tout au moins des moyens les plus efficaces de repérer l'information et de l'obtenir dans des délais raisonnables. La Bibliothèque se devait donc de mettre de l'avant une stratégie permettant à nos usagers de contourner les contraintes associées à notre éloignement et à notre statut d'université de taille modeste, sans quoi la survie de l'UQAC elle-même comme établissement universitaire de qualité se trouverait menacée.

Jusque-là, nous direz-vous, rien de bien original. Ce constat s'applique sans doute aujourd'hui à la majorité des bibliothèques universitaires en Amérique du nord. Les réflexions qui découlaient de ce constat devaient nous amener très loin. En toute logique, si le constat était fondé, il imposait une grande priorité: tout mettre en oeuvre pour optimiser la capacité d'information du système-bibliothèque dans le cadre d'un fonctionnement totalement et essentiellement dédié à la satisfaction des besoins dinformation de nos usagers. Nous pourrions disserter longuement sur la portée de cet énoncé qui contient en luimême les germes de la révolution organisationnelle qu'a connu notre Bibliothèque. Nous avons d'ailleurs eul'occasion d'aborder cet aspect dans d'autres textes ${ }^{5}$.

\section{Les orientations proposées}

La démarche de réflexion enclenchée devait trouver sa concrétisation dans l'énoncé d'orientations (annexe 1) qui, depuis douze ans, sert de guide et de grille d'interprétation à toutes nos actions. À la Bibliothèque, cet énoncé qui tient sur une page fait figure de texte quasi messianique. Commentons-en brièvement les grandes lignes.

D'abord, priorité au développement des moyens permettant d'optimiser notre capacité d'information. Ceci implique la présence de personnels compétents disposant des facilités techniques appropriées et évoluant dans un contexte physique et organisationnel favorisant l'acquisition de savoir-faire de haut niveau. Le complément nécessaire à ce choix stratégique nous imposait de faire connaître et partager par nos clients ce savoir-faire, d'où l'importance accordée à la Bibliothèque de I'UQAC à la formation à l'information des usagers. S'il est vrai que l'accès à une information de qualité constitue un préalable incontournable à la compétitivité des organisations et des individus, encore fautil que ces derniers puissent utiliser efficacement les possibilités mises à leur disposition. À cette fin, il faut que la formation à l'information de nos étudiants devienne un élément essentiel de leur formation de base.

Secundo, la bibliothèque doit développer un fonds documentaire local susceptible de satisfaire aux besoins des étudiants de premier cycle, d'appuyer la priorité précédente (accès à l'information) et de constituer, dans des secteurs très précis identifiés aux axes de développement de l'Université, des collections en mesure de satisfaire les besoins des étudiants des $2 e$ et $3 e$ cycles et des chercheurs. Le tout devant s'appuyer sur un système efficace de fourniture de documents.

Tertio, la Bibliothèque doit rendre disponibles ses services à la communauté régionale.

Une fois acquis que la Bibliothèque de I'UQAC trouverait sa justification premièrenon dans l'acquisition de la documention mais dans sa capacité à rendre disponible l'information nécessaire à la satisfaction des besoins de ses usagers, tout était remis en cause et nombre de questions se posaient. Par exemple, le modèle organisationnel proposé par la bibliothèque traditionnelle et orienté vers la mise à la disposition du client de la documentation, et non de l'information, offre-t-il une performance optimale eu égard aux orientations qui devenaient les nôtres? Le type de personnel recruté, rompu aux techniques bibliothéconomiques, est-il en mesure de répondre aux attentes nouvelles fondées non plus sur l'application de techniques documentaires mais sur le développe- ment de connaissances en information de haut niveau? Notre propre gestion qui s'appuie sur un modèle tayloriste, somme toute rassurant, est-elle à la hauteur d'objectifs qui empruntent davantage à la gestion de l'intelligence qu'à celle d'objets physiques (documents de toute nature) ou d'humains robotisés? Bref, les implications des choix stratégiques que nous avions faits étaient colossales et nous ne disposions à l'époque que de bien peu d'exemples pour nous conforter dans nos choix. Nous avons tenté de répondre à ces interrogations en élaborant un modèle organisationnel nouveau qui fera l'objet de la brève description qui suit.

\section{Le modèle organisationnel}

Sur le plan structurel, la Bibliothèque del'UQAC compte, outre la direction, deux grandes divisions: la Division des services de gestion documentaire et la Division des services d'information documentaire (voir tableau à la page suivante). Là s'arrêtent cependant les similitudes avec les structures traditionnelles dans nos bibliothèques.

Compte tenu du fait que notre grande priorité est la mise à la disposition du client de l'information, et non de la documentation, que l'information est de nature essentiellement disciplinaire, qu'elle impose ses lois et contraintes qui, par nature, n'ont rien à voir avec celles associées au document (par exemple, l'information vieillit rapidement, elle a tendance à se spécialiser, elle est de qualité variable, etc.), il fallait que le modèle organisationnel tienne compte des contraintes associées à notre objet, objet qui n'est pas un élément physique (livre) ou un édifice (la bibliothèque), mais un hybride, à la fois physique et intangible, l'information enregistrée. Ceci devait nécessairement se refléter dans la structure en place. Nous

5. Gilles Caron, «Vers une philosophie de la bibliothéconomie», Documentation et bibliothèques, vol. 21, no 4 (décembre 1975), 187-196.

- «Bibliothécaire ou administrateur: un choix à faire?», Argus, vol. 6, no 5 (sept-oct. 1977), 7882.

Gilles Caron et Richard Boivin, «Le bibliothécaire, la bibliothèque et le changement», Argus, vol. 9, no 2 (mars-avril 1980), 53-56. 
Tableau : Structure matricielle de la Bibliothèque de I'Université de Chicoutimi

\section{Fonctions}

$\begin{aligned} \text { 1. } & \text { Acquisitions } \\ \text { 2. } & \text { SIGIRD } \\ \text { 3. } & \text { CD-ROM } \\ \text { 4. } & \text { Choix } \\ \text { 5. } & \text { Comptabilité documentaire } \\ 6 . & \text { Dons \& échanges } \\ \text { 7. } & \text { Formation documentaire } \\ \text { 8. } & \text { Livres rares } \\ \text { 9. } & \text { Prêt entre bibliothèques (PEB) } \\ \text { 10. } & \text { Traitement matériel } \\ \text { 11. } & \text { Prêt } \\ \text { 12. } & \text { Rayonnage } \\ \text { 13. } & \text { Information/communication } \\ \text { 14. } & \text { Référence ( } r \text { ) } \\ \text { 15. } & \text { Téléréférence }\end{aligned}$

15.1 Bases de données bibliographiques

15.2 Bases de données numériques

15.3 Autres (INTERNET, etc.)

16. Traitement documentaire

16.1 Indexation-classification

16.2 Catalogage

16.3 Inventaires

16.4 Pré-catalogage

17. Vérification bibliographique



$\mid$

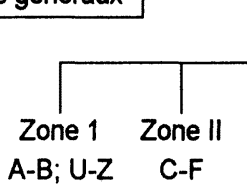

Division des services d'information documentaire

Zone 1 Zone II Zone III Zone IV Zone V Zone VI Zone VII Zone VIII Zone IX

A-B; U-Z C-F G: Carto- Ét. rég. Pub. off. H-K L-P Q-T Audiovidéo theque theque

$r$ = L'animation est assurée par le responsable de la Division.

$E=$ Fonction exclusive à l'une ou l'autre division

$\mathrm{P}=$ Fonction assurée par les deux divisions

$X d=$ Localisation physique de la personne en charge d'une fonction donnée

$m=$ Participation marginale

Ét. rég. = Études régionales

Publ. off. = Publications officielles

devions passer d'un modèle organisationnel performant à rendre disponible la documentation au client à un autre dont l'efficacité serait mesurée à sa capacité de satisfaire aux attentes en information de ce dernier.

Le modèle proposé et la structure matricielle qui le sous-tend devaient répondre à ces attentes.

En pratique, la Bibliothèque compte toujours deux divisions. La Division des services d'information documentaire regroupe tous les employés qui s'identifient aux secteurs disciplinaires ou disciplines représentés à l'UQAC. Dans le cadre de leur travail, les employés de cette division exercent un certain nombre de fonctions susceptibles de contribuer à l'enrichissement de leur connaissance disciplinaire et, par delà, à la satisfaction des besoins d'information de leurs clients. Par exemple, le choix, la référence, la formation documentaire des usagers, l'exploitation des bases de données et le traitement documentaire sont, à I'UQAC, des fonctions qui se trouvent dans une large mesure monopolisées par la Division des services d'information documentaire. La Division des services de gestion documentaire regroupe par contre toutes les fonctions sans connotations disciplinaires particulières. C'est le cas des acquisitions, de la préparation matérielle, du rayonnage, du prêt de la documentation, etc. 
Tous les professionnels et la plus grande partie des techniciens se retrouvent dans la Division des services d'information documentaire, l'autre division comptant principalement des commis. Les professionnels et techniciens de la Division des services d'information documentaire travaillent en équipe restreinte (professionnel et technicien), chaque équipe s'identifiant à une ou quelques disciplines appelées "secteur» et à un espace physique appelé «zone» ou «îlot».

II n'y a plus à la Bibliothèque de I'UQAC de responsables hiérarchiques qui s'identifient à une fonction spécifique (ex.: responsable du traitement, des acquisitions, de la référence, etc.), mais des responsables de dossier qui, dans le cadre d'une structure matricielle privilégiant la discipline, sont chargés de l'animation et du développement de la fonction qui leur est impartie. Ces responsabilités de fonction incombent généralement à l'un ou l'autre des professionnels de la Division des services đ'information documentaire (voir tableau). Ainsi, le professionnel du secteur éducation-psychologie, identifié à la zone éducation-lettres (zone VII) est aussi le coordonnateur de l'animation et du développement de la fonction uformation des usagers». II sert de support à ses confrères pour tous les aspects reliés aux modèles pédagogiques à utiliser; il se tient au fait des expériences réalisées ailleurs; il supervise le développement des outils d'apprentissage et suggère à son supérieur immédiat; le responsable de la Division des services d'information documentaire, les orientations et décisions à mettre de l'avant. II en est de même pour la référence automatisée, les banques de données numériques, le traitement documentaire, l'élagage, etc., bref, toutes ces fonctions traditionnelles que l'on retrouve dans l'ensemble des bibliothèques.

\section{L'impact des orientations et du modèle organisationnel}

\section{L'impact sur les aménagements physi-} ques $^{6}$

La première chose qui frappe les personnes qui visitent notre Bibliothèque, c'est la dispersion physique du personnel. Le fonctionnement en zones qui recoupent grosso modo la nomenclature des cotes du Congrès fait en sorte que le personnel se retrouve déployé sur l'ensemble de la surface de la Bibliothèque. On retrouve des employés partout!... Ce qui n'est pas sans occasionner parfois des «problèmes d'images". II n'y a plus d'espace physique réservé au traitement documentaire, lequel s'effectue désormais dans les zones.

Une des conséquences voulues du modèle était de maximiser le pourcentage de nos employés en contact direct et quotidien avec les clients. A la Bibliothèque de I'UQAC, à part les cinq membres de la direction (le directeur, les deux adjoints et les deux secrétaires), seules sept personnes sur 41 ne sont pas en contact direct permanent avec les usagers. Ce seul fait a eu un impact considérable sur la vision "service» que nous voulions être l'image de marque de la Bibliothèque. Pour la quasi-totalité de notre personnel, l'usager n'est plus une abstraction; c'est une personne réelle qui a des besoins bien concrets et qui, du seul fait de sa présence, exerce une pression considérable sur lui. Autre effet significatif, il n'y a plus de tiraillements entre les deux divisions, tiraillements qui sont omniprésents dans le modèle traditionnel où chaque grande division (services techniques et services au public) possède sa culture propre et sa vision souvent différentes, sinon conflictuelles, du service à offrir. À I'UQAC, les employés des deux divisions vivent ce contact permanent avec l'usager ce qui a pour effet de modifier radicalement la perspective.

\section{L'impact sur le fonctionnement courant.}

Plusieurs éléments pourraient être soulignés ici. Contentons-nous d'en noter deux:

\section{Le fonctionnement dans le cadre de la structure matricielle.}

Ceux qui ont fait l'expérience d'une structure matricielle vous diront que le grand défi se situe dans le maintien de l'équilibre, d'une part, entre les individus ou groupes afin de maintenir l'équilibre des tâches entre eux et, d'autre part, au sein de chaque unité, entre les préoccupations disciplinaires et fonctionnelles. Ce dernier point mérite une attention toute particulière. Par exemple, la personne qui joue un rôle à la fois comme responsable de discipline et animateur de fonction sera confrontée en permanence avec des choix douloureux associés à l'utilisation de son temps. La gestion du temps exige des personnes qui oeuvrent dans ce cadre une discipline rigoureuse, d'autant plus que les niveaux d'intervention sont différents. À titre de conseiller disciplinaire, l'employé est généralement confronté à un usager externe (étudiant ou professeur) qui exige une réponse immédiate à ses besoins. Dans le cadre de son rôle fonctionnel, il offre un service à l'organisation (ses usagers étant ses collègues) dans une perspective souvent associée au développement de la fonction et qui implique des interventions dont les incidences se manifesteront à moyen et long termes.

Puisque le «leitmotiv» du service veut que tout doit viser les intérêts des usagers, que la pression de ces derniers est permanente, le rôle fonctionnel risque d'en souffrir au point de pénaliser les intérêts à moyen terme de ce même usager et de l'ensemble du Service. C'est d'ailleurs là que se situe le coeur du rôle dévolu au responsable de la Division des services d'information documentaire. $\mathrm{Ce}$ dernier, à la fois animateur-coordonnateur et responsable hiérarchique doit jongler en permanence avec les intérêts à court, moyen et long termes des usagers. Ceci l'oblige à une vision d'ensemble cohérente. Sur le plan de la gestion, il doit maintenir, sinon imposer à ses troupes, l'équilibre nécessaire entre leurs tâches de façon à faire en sorte que, non seulement le service soit de qualité aujourd'hui, mais le demeure demain.

Les interventions du coordonnateur s'exercent généralement au cours des exercices de planification annuelle où chaque responsable de secteur doit faire état de ses projets dans le cadre du double mandat qui est le sien. Tout cela doit se réaliser dans le respect à la fois de l'autonomie réelle dont jouit chaque secteur dans la définition de ses priorités et de l'obligation des gestionnaires de voir au développement harmonieux de l'ensemble.

6. Voir, en annexe 2, l'aménagement d'une zone. 


\section{L'organisation des chaînes de travail}

L'aspect technique de l'organisation des chaînes de travail, en particulier au titre du traitement, n'a pas dans l'ensemble fait problème. Comme le souligne à juste titre Martell? , les moyens technologiques dont nous disposons actuellement permettent de contourner les obstacles qui imposaient auparavant la centralisation des activités. Bien sûr, la manipulation des documents s'en est trouvée accrue. Par contre, notre mode de fonctionnement a permis d'augmenter la disponibilité physique réelle des documents dans la zone, et ce, dès réception, un service à valeur ajoutée qui compense largement les désagréments associés à la manipulation additionnelle des documents. Des efforts d'adaptation ont dû par contre se faire au niveau de la gestion quotidienne et du contrôle de production, lesquels ont dû être repensés complètement. Nous aborderons cet aspect ultérieurement.

\section{L'impact sur les politiques de gestion du personnel}

\section{Les professionnels}

Sile modèle présenté ici est en place depuis juin 1982, il n'en était pas moins en gestation à la Bibliothèque depuis un bon moment déjà. À partir des années 1975, les professionnels de secteur, dans la structure traditionnelle, participaient à la fois au service au public et au traitement de la documentation. Dès cette époque s'est posée la question d'une politique de recrutement et de sélection en accord avec le tournant que nous anticipions.

En 1979, nous définissions les qualifications minimales attendues des professionnels qui aspiraient à travailler à notre Bibliothèque. Très vite le consensus s'est établi sur le fait qu'en toute occasion, la spécialisation disciplinaire devait être considérée comme incontournable et qu'elle devait être du plus haut niveau possible. De façon concrète, ceci voulait dire que nous recrutions des professionnels qui disposaient d'une formation disciplinaire poussée et, si possible, d'une formation en bibliothéconomie en plus. Ceci voulait dire aussi en corollaire que, si nous ne pouvions identifier des candidats valables disposant d'une double formation, nous privilégions la formation disciplinaire. Aujourd'hui, sur les huit professionnels que compte la Division des services d'information documentaire, quatre ne sont pas bibliothécaires de formation. Deux sont ingénieurs (maîtrise en génie chimique et géologie), l'un est un diplômé en mathématique et le dernier possède un doctorat en lettres. Ces personnes se sont très bien intégrées à notre système.

\section{Les bibliotechniciens}

Pour la quasi-totalité des bibliotechniciens de la Bibliothèque, la nouvelle structure représentait un bouleversement total. Plus encore que pour les professionnels, leur environnement fut modifié considérablement. Et c'est d'ailleurs de ce groupe d'employés que parvinrent les réactions les plus vives à la nouvelle structure. Et pour cause! Du jour au lendemain, nous demandions à une personne qui avait 10 ans d'expérience en catalogage de travailler désormais avec un professionnel dans un secteur disciplinaire précis où l'éventail des tâches incluait la référence de premier niveau, le catalogage, la participation aux activités de formation des usagers, etc., bref un éventail somme toute comparable à celui du professionnel mais à un palier différent. Pour cette raison, l'opération démarrage fut laborieuse. Aujourd'hui, nous pourrions mettre au défi quiconque pourrait identifier un de nos bibliotechniciens qui souhaiterait revenir en arrière.

\section{Les commis}

La situation des commis, dans l'ensemble, ne fut pas sensiblement modifiée par l'implantation de la nouvelle structure. Même si l'enrichissement des tâches du personnel n'était pas un des objectifs recherchés par l'implantation de la nouvelle structure, ce n'en fut pas moins une des conséquences indirectes du modèle que nous implantions. Nos commis, qui dans un premier temps s'étaient sentis à l'abri des «affres du changement» imposé à leurs collègues, considèrent aujourd'hui pour certains avoir été laissés pour compte dans cette opération. Cette situation nous préoccupe aujourd'hui et nous obligera à intervenir à court et moyen termes.

\section{L'impact sur les pratiques de gestion}

Le modèle de gestion en place à I'UQAC, on s'en doutera, est éminemment décentralisé en ce qui a trait au fonctionnement. C'est le personnel directement au service des clients qui assume la responsabilité quotidienne totale quant aux «quoi» et aux «comment».

Le rôle de la gestion est double: d'une part, support et animation de l'ensemble et, d'autre part, chien de garde des intérêts collectifs, en particulier au niveau de l'allocation des ressources et des règles de fonctionnement minimales à respecter. Plusieurs aspects de la philosophie de gestion qui nous animent pourraient être soulevés ici. Notons-en deux au passage:

\section{La gestion des priorités}

Les responsables de secteurs fixent leurs priorités; la Bibliothèque fixe les siennes et l'Institution également. Le rôle de la gestion est d'harmoniser le tout de façon à produire un plan annuel de travail cohérent autour duquel tous se retrouvent. Nous le soulignions, au sein de la zone, les membres du personnel en place sont responsables, selon leur perception des intérêts supérieurs de l'usager, de définir ce qu'ils feront et quand ils le feront. Ceci a pour effet de mener à des fluctuations importantes de «l'output» de telle ou telle fonction selon les périodes de l'année. À titre d'exemple, les débuts d'année universitaire étant largement monopolisés par l'accueil et la formation des étudiants, le nombre de documents traités peut chuter considérablement pendant cette période. Par contre, les fins de trimestre seront généralement extrêmement productives à ce titre. Ceci n'est pas sans imposer aux gestionnaires des façons très différentes de gérer.

Les mesures de contrôle et évaluation

Compte tenu de ce qui précède, on aura compris que les procédures de contrôle des extrants devront être adaptées au vécu de fonctionnement. À l'UQAC, les statistiques de production prennent un

7. Charles Martell, The Client..., p. 69. 
sens lorsqu'on les observe sur une base trimestrielle. II demeure hasardeux, en effet, d'extrapoler sur les tendances de l'année à partir des statistiques hebdomadaires ou même mensuelles. Le prix à payer d'un tel état de fait est que notre capacité de réaction comme gestionnaire s'exerce davantage en différé qu'en temps réel. L'insécurité qu'une telle situation pourrait créer est cependant grandement atténuée par l'importance accordée à une circulation extrêmement libérale de l'information. Nous avons en effet mis en place des mécanismes de transfert de l'information dans les deux sens susceptibles de nous permettre en tout temps de «jauger» des actions en cours dans le service.

L'évaluation de la qualité globale du service fait l'objet actuellement d'une attention soutenue. Si, en effet, notre fonctionnement est très décentralisé, s'il est vrai que nos gens jouissent d'une latitude considérable leur permettant de définir ce qui doit être fait, quand et, dans une large mesure, comment, il faut en contrepartie que nous disposions, sur le plan de la gestion, des moyens d'apprécier le service offert tant sur le plan qualitatif que quantitatif. C'est là une question de responsabilité fondamentale des gestionnaires dans lecadre de leur mandat. Outre les statistiques et rapports annuels de circonstance, cette évaluation s'exerce prioritairement auprès des usagers au moyen de deux mécanismes principaux: le Comité de la Bibliothèque et éventuellement, l'enquête annuelle auprès des usagers.

Notre Comité de la Bibliothèque est actif depuis plus de 15 ans. II regroupe un représentant-professeur de chacun des huit départements, deux représentantsétudiants des programmes des $2^{e}$ et $3^{e}$ cycles et de premier cycle ainsi que les doyens du premier cycle et des études avancées et de la recherche. Le Comité se réunit trois fois l'an et on $y$ aborde les questions d'intérêt général susceptibles de toucher nos usagers. Depuis maintenant trois ans, des sous-comités sont créés de façon «ad hoc» pour étudier des problèmes précis et soumettre des recommandations au Comité général et au directeur. Le "feedback» que nous recevons du Comité de la Bibliothèque est hautement significatif et très apprécié.
Parallèlement, nous sommes actuellement à étudier les moyens de mettre en place un mécanisme d'enquête annuelle auprès de nos clients. Ce projet se poursuit et nous espérons pouvoir procéder d'ici les deux prochaines années.

\section{La zone disciplinaire type}

\section{Aspect physique}

L'annexe 2 illustre graphiquement ce à quoi ressemble, à I'UQAC, une zone disciplinaire. En pratique, on trouve regroupé, dans un espace physique donné, l'ensemble de ressources qui permettent à l'usager de s'approprier la quasi-totalité des services susceptibles de satisfaire ses besoins d'information dans sa discipline. Autour des bureaux du professionnel et du technicien, on retrouve les ouvrages de référence pertinents au secteur, des terminaux servant à l'interrogation des divers fichiers, la collection du secteur, des tables de travail et des salles de travail de groupes pour consultation de la documentation ainsi que des étagères regroupant les acquisitions récentes en cours de traitement. Dans la mesure du possible, nous essayons de faire en sorte que l'usager puisse s'identifier à la fois à des employés et à un espace physique bien identifié au sein de la Bibliothèque. Les employés des zones ainsi constituées sont encouragés à donner un cachet caractéristique à leur environnement, à refléter, tant sur le plan de l'aspect physique que des services offerts, la "culture» de la ou des disciplines desservies. C'est ainsi que des services seront offerts dans une zone et non dans une autre, que certaines façons de faire seront différentes et que même l'aménagement physique pourra varier.

\section{Le fonctionnement courant}

La définition des priorités quotidiennes est laissée entièrement entre les mains du personnel de la zone qui, en contact direct et permanent avec les étudiants et les professeurs, est le plus en mesure de les préciser. Chacun, le professionnel et le technicien, dans le cadre des limites de leurs interventions, exécute l'une ou l'autre des fonctions prioritairement requises par les usagers. Les interactions entre professionnels et techniciens ne font généralement pas problème, les uns et les autres se référant habituellement les usagers selon leurs compétences respectives.

Les échanges entre zones sont permanents. Ils se fondent soit sur le partage "ad hoc» de connaissances ou d'interrogations face à des problèmes quotidiens liés à des fonctions précises (ex.: la téléréférence, le traitement documentaire, la formation documentaire, etc.), soit sur des échanges plus formels entre employés de niveaux différents associés dans le cadre de mandats spécifiques (ex.: le programme d'information-communication, le Comité Sigird local, le programme de développement du personnel, etc.).

De façon générale, même si des personnes assument des responsabilités fonctionnelles, nous essayons de faire en sorte que nos actions et comportements imposent la solidarité collective comme valeur fondamentale au sein de l'organisation. Les problèmes d'un tel ou d'une telle dans l'une ou l'autre des zones ou divisions sont susceptibles d'affecter tout le monde dans le Service. Chacun doit en prendre conscience et se montrer disposé, lorsque faire se peut, à apporter un appui tangible à leur résolution. L'ensemble du personnel, par exemple, est habitué à nos «blitz» annuels qui, autour d'une situation donnée, réunissent tout le monde du directeur au «rayonneur» dans des opérations de reprise en main d'un aspect ou l'autre au sein de l'organisation.

Si nous devions, par analogie, comparer notre modèle de fonctionnement par zone à quelque chose, c'est le modèle de la PME (petite et moyenne entreprise) qui s'imposerait. La Bibliothèque de I'UQAC est un regroupement de PME qui poursuivent au sein d'un ensemble plus large en mesure de les appuyer des objectifs associés à la fourniture de services d'information de qualité.

\section{Les contraintes imposées par le modè- le}

Sur le plan de la gestion, outre une adaptation nécessaire des gestionnaires à la réalité d'une décentralisation réelle des pouvoirs et responsabilités et peutêtre précisément à cause de cet état de fait, le modèle en place impose certaines contraintes difficilement contournables. 
Comme nous y faisions mention un peu plus haut, la nécessité d'indicateurs externes probants constitue un impératif essentiel. D'autant plus que notre produit n'est plus un élément physique (document), mais un élément intangible (l'information à l'usager) dont l'évaluation de performance comme service est de nature éminemment subjective. Nous consacrons actuellement beaucoup d'efforts afin de développer des indicateurs qui reflètent les finalités poursuivies, un travail délicat dont la complexité a été reconnue par d'autres au sein de la profession ${ }^{8}$.

Entre temps, nous accordons une attention extrême à notre Comité de la Bibliothèque dont les avis et recommandations sont d'un précieux secours. Parallèlement, nous intensifions les efforts visant à intégrer au maximum les préoccupations relatives aux services d'information à offrir dans le cadre des instances reconnues de l'UQAC. Nous y reviendrons plus loin.

Une autre importante contrainte du système en place tient à la quasi-absence de mobilité des employés d'une zone à une autre zone. La priorité accordée à la compétence disciplinaire a fait en sorte de priver une grande partie des employés de toute forme de polyvalence. Cette absence de polyvalence est totale chez nos professionnels, importante dans le cas des techniciens qui se sentent de plus en plus limités hors de leur champ disciplinaire respectif. Cette situation n'est pas sans nous préoccuper et peu de solutions sont envisagées actuellement.

\section{Les résultats obtenus}

Nous ne disposons pas actuellement de données scientifiques véritables permettant de porter un jugement définitif sur la performance du modèle mis en place. Tout au plus disposons-nous d'indications ou de témoignages souvent indirects susceptibles de nous rassurer et peut-être même de nous laisser percevoir que le modèle satisfait aux attentes pressenties, c'est-à-dire l'optimisation de la capacité d'information du système au bénéfice des usagers. Notons tout de même au passage certains indices. L'image de la Bibliothèque sur le campus est généralement excellente comme en font foi tant les sondages internes ${ }^{9}$ réalisés au cours des dernières années auprès des ex-étudiants que les sondages externes ${ }^{10}$. La performance de nos chercheurs se situe parmi les meilleures au Québec. II n'existe encore aucun centre de documentation départemental sur le campus et la crédibilité de la Bibliothèque tant auprès des usagers que des administrateurs de l'Université est excellente. La Bibliothèque a su, au cours des ans, développer des services originaux (pensons au programme de formation documentaire crédité) qui la situent à l'avant-garde au Québec.

\section{Les coûts}

Les orientations que nous nous sommes données et la structure en place qui vient les appuyer sont neutres quant aux coûts. Le "modèle UQAC» ne coûte pas plus cher que le modèle traditionnel. Par exemple, le budget de la Bibliothèque en 1993-94 se situera à environ 5,9\% du budget de l'établissement. Nous espérons d'ici deux ans porter cette proportion à $6 \%$ et, sur cette base, consacrer à l'acquisition de la documentation $25 \%$ du budget global de la Bibliothèque. Ces deux rapports nous situeront globalement dans la moyenne québécoise pour les établissements comparables.

Le modèle UQAC, rappelons-le, vise à accroître la capacité d'information du système bibliothèque. C'est un modèle essentiellement qualitatif. De ce fait, il n'implique et n'impose aucun déplacement de ressources de la documentation vers l'information ou vice-versa. Ce point est extrêmement important à souligner auprès des administrateurs supérieurs et de certaines clientèles qui, confrontés à la pauvreté grandissante de nos fonds documentaires, pourraienty percevoir un moyen additionnel des "gens de la Bibliothèque» pour les priver d'une documentation qui déjà leur fait cruellement défaut.

\section{Les développements à venir}

\section{Sur le plan intra-organisationnel}

Trois éléments retiendront notre attention au cours des prochaines années. D'abord, nous devrons compléter l'intégration des collections spéciales que sont l'audiovidéothèque et les publications officielles auxzones disciplinaires. Ces deux collections demeurent à I'UQAC des enti- tés physiques séparées. Pour des raisons compréhensibles, elles le demeureront probablement. Cependant, l'intégration des contenus aux zones disciplinaires devra dépasser le niveau du catalogue collectif commun qui demeure un acquis minimal. Au même titre que tout autre document de la Bibliothèque, les collections de ces deux entités doivent faire partie du bagage d'informations de notre personnel de zone. Nous avons mis en place diverses mesures pour ce faire.

Secundo, la responsabilité des personnels de zones dans le développement et le support aux dossiers de fonction devra être renforcée. Comme nous le soulignions précédemment, l'équilibre entre les rôles disciplinaires et fonctionnels demeure fragile. Des actions plus rigoureuses devront être entreprises afin de bien ancrer ces deux niveaux de responsabilité dans la tâche de chacun.

Tertio, nous devrons mettre en place un programme cohérent de formation et de perfectionnement de notre personnel sous la gouverne de la Bibliothèque. Jusqu'à maintenant, la plupart des activités en la matière ont été laissées à l'initiative des individus et se sont exercées au sein des divers comités conjoints syndicatspatrons de l'établissement. Nous considérons, attendu les défis associés entre autres à l'implantation des nouvelles technologies, que la Bibliothèque doive assumer une responsabilité accentuée dans ce dossier.

8. Doris J. Schlichter and Michael J. Pemberton, "The Emperor's new clothes? Problems of the user survey as a planning tool in academic libraries», College \& Research Libraries, vol. 53 , no. 3 (May 1992), 257-265.

9. Enquête-sondage sur le niveau de satisfaction des étudiants de l'UQAC, projet RELANCE 1987, Satisfaction à l'égard des services, Chicoutimi, UQAC-Service des communications institutionnelles, 1987. (Voir grille synthèse en annexe 4)

10. Sondage CAMPUS-COORS mené auprès de 2400 étudiants inscrits au premier cycle dans les universités du Québec. Le seul endroit du sondage où l'UQAC se situait en tête concerne "Les outils de travail») (équipement audiovisuel, bibliothèque, etc.), Groupe Everest Inc., secteur SEROM, mai 1987. 


\section{Sur le plan institutionnel}

Tous les efforts actuels tendent vers une imbrication optimale de la Bibliothèque aux besoins et préoccupations de l'enseignement et de la recherche. Cette perméabilité se concrétisera à deux niveaux:

\section{Sur le plan opérationnel}

La formation à l'information des usagers, nous le soulignions, tient, depuis plus de quinze ans, une grande importance. De plus en plus orientées vers le cours crédité, de telles actions se sont jusqu'à aujourd'hui réalisées sur des bases généralement informelles à la suite de demandes de professeurs ou de responsables de programmes ou modules qui considéraient ces activités indispensables à leurs étudiants. Celles-ci, rappelons-le, touchent aujourd'hui plus de $60 \%$ des nouveaux étudiants inscrits à l'UQAC.

Nous pensons qu'il est désormais temps d'envisager une nouvelle étape. $\AA$ l'hiver 1993, le Décanat des études de premier cycle et le Service de la bibliothèque mettaient en place un groupe de travail conjoint chargé de proposer un cadre conceptuel et fonctionnel permettant l'institutionnalisation d'une activité de formation documentaire crédité obligatoire dans tous les programmes de premier cycle. Le groupe de travail doit remettre un rapport préliminaire en septembre 1994.

\section{Sur le plan formel}

Nous avons entrepris des démarches visant à accentuer la présence de la Bibliothèque au sein des instances d'enseignement et de recherche afin de faire en sorte que les préoccupations et attentes des uns et des autres soient mieux comprises et coordonnées. À cet égard, nous encourageons nos professionnels à participer aux activités des conseils de module; nous avons suggéré aux autorités de s'assurer de la présence de responsables de la Bibliothèque au sein des sous-commissions des études de premier cycle et des études avancées et de la recherche. Par ailleurs, les doyens des études de premier cycle et des études avancées et de la recherche sont membres "de facto» du Comité de la bibliothèque. Toutes ces mesures, croyons-nous, sont de nature à permettre l'harmonisation et l'intégration optimale du Service de la bibliothèque avec l'enseignement et la recherche.

\section{Sur le plan régional}

La Bibliothèque de I'UQAC souhaite rendre disponible son savoir-faire et ses capacités à l'ensemble de la région. Nous avons produit au printemps 1992 un plan d'intervention élargi en région qui inclut, entre autres choses, l'aide à la mise en place d'un service d'information en appui au développement des entreprises locales. Nous sommes actuellement à évaluer la demande réelle pour un tel service.

\section{Le modèle de I'UQAC est-il «exporta- ble»?}

La Bibliothèque de I'UQAC est et demeurera une bibliothèque universitaire d'envergure modeste. Les professionnels de l'information documentaire que nous recevons nous interrogent inévitablement sur les possibilités de transposer, en tout ou en partie, le modèle en place dans une bibliothèque plus importante. Un tel modèle est-il possible et souhaitable dans une grande bibliothèque universitaire et, si oui, produirait-il les mêmes retombées?

À la Bibliothèque de l'UQAC, nous sommes persuadés que le modèle est transposable dans tout organisme qui tire sa raison d'être du traitement et de la mise à la disposition des usagers de produits d'information spécialisés. Qui plus est, nous sommes convaincus que les avantages attendus d'un tel modèle ne pourront que croître selon un rapport directement proportionnel à l'importance des équipes en place. En effet, la présence d'un personnel plus nombreux devrait permettre à une organisation type de spécialiser davantage le savoir-faire disciplinaire, d'où l'obtention de services de qualité accrue. Plus le champ disciplinaire à couvrir sera étroit, meilleur sera le niveau de connaissance atteint et, conséquemment, si les finalités sont bien comprises, le niveau de service à l'usager.

\section{Et la technologie?}

Au même titre que pour les modes associées à la gestion administrative et financière, la technologie demeure pour nous un moyen, non une fin. Élémentaire, direz-vous. Cependant, force est de reconnaître que les moyens ont souvent, dans notre profession, transcendé les finalités. Cette fixation sur les moyens que manifestent beaucoup de professionnels au sein de notre profession, aura probablement été un des obstacles majeurs à une redéfinition en profondeur des systèmes documentaires. L'arrivée de l'informatique, par exemple, a permis de perpétuer, par un ajout d'efficacité certain, des façons de faire dont la pertinence aurait probablement dû être remise en question depuis fort longtemps ${ }^{11}$. Pour beaucoup, elle aura de fait constitué un frein au changement.

Cela dit, nous ne voudrions pas laisser le lecteur sur l'impression que nous ne nous préoccupons pas à I'UQAC d'automatisation et d'accès tous azimuts à l'information disponible. Bien au contraire. La Bibliothèque de I'UQAC attache une attention soutenue à l'intégration des moyens techniques les plus modernes. Cependant, nous sommes convaincus que les véritables enjeux au sein de notre profession ne sont pas de nature technique ou technologique. Ils sont de nature beaucoup plus fondamentaux. Ils portent, selon nous, sur notre objet et sur les finalités qui sont les nôtres à titre de spécialiste de l'information documentaire. II y a là les éléments de base d'une réflexion qui demeure encore largement à faire au sein de la profession.

\section{Conclusion}

La philosophie de gestion qui est la nôtre, les orientations que nous nous sommes données et le modèle organisationnel en place ont, croyons-nous, démontré sinon leur pertinence du moins leur faisabilité. Ils ont permis à la Bibliothèque de I'UQAC d'offrir à ses usagers des services à valeur ajoutée digne des meilleurs organismes d'information documentaire et d'imposer par là sa crédibilité. La Bibliothèque dispose maintenant de moyens et savoir-faire spécifiques qui la positionnent avantageusement face aux défis que pose aux étudiants et aux chercheurs la mise en place de la société dite d'information.

11. Gilles Caron et Richard Boivin, «Le bibliothécaire... 


\section{Annexe 1: Les orientations du service de la bibliothèque*}

Compte tenu :

A) De la mission du Service de la bibliothèque :

- support à l'enseignement et à la recherche

- support à la collectivité régionale

B) De la problématique du développement de la Bibliothèque, à savoir:

1. les conditions objectives imposées à son développement:

- financement limité

- isolement géographique

- faible collection locale

- importance grandissante des études avancées et de la recherche

- variété des programmes disciplinaires

2. la situation présente et l'évolution prévisible du secteur information/documentation:

- croissance soutenue du volume documentaire

- multiplicité et complexité grandissantes des modes d'accès et supports

- révolution télématique

3. les moyens et possibilités de la Bibliothèque (expertise développée):

- dans l'exploitation des systèmes de repérage de l'information (banques de données)

- dans la formation des usagers

- dans l'automatisation interne (BADADUQ)

La Bibliothèque retient les orientations suivantes comme lignes directrices de son développement:

A) au niveau de l'accès à l'information:

1. La Bibliothèque de l'UQAC doit rendre disponible à ses usagers, particulièrement à ses chercheurs et étudiants gradués, l'information nécessaire à leurs besoins, grâce à la mise en place de mécanismes adéquats d'accès à l'information.

2. La Bibliothèque de l'UQAC doit assurer à ses usagers une formation minimale à l'information qui leur permette:

a) comme professionnel, d'acquérir les bases d'un fonctionnement autonome quant à la satisfaction de leurs besoins d'information dans leur discipline;

b) comme étudiant, d'exploiter les sources d'information mises à leur disposition.

B) au niveau de l'accès à la documentation:

1. La Bibliothèque de I'UQAC doit rendre disponibles à ses usagers:

a) une collection pertinente aux axes de développement institutionnel, à savoir le Moyen-Nord et les Études et interventions régionales;

b) un large éventail des outils (manuels, ouvrages de référence, etc.) nécessaires au repérage de l'information;

c) une collection fonctionnelle de documents de base pertinents aux programmes dispensés à l'UQAC

2. La Bibliothèque de l'UQAC doit rendre disponibles à ses usagers les mécanismes d'accès à la documentation extérieure les plus adéquats (ex.: PEB)

C) au niveau de l'implication régionale :

La Bibliothèque de l'UQAC rend disponibles à la population régionale ses ressources et son expertise, dans les limites de ses moyens.

* Approuvées par le Conseil d'administration de l'UQAC le 30 juin 1982. 
Annexe 2: La zone disciplinaire type
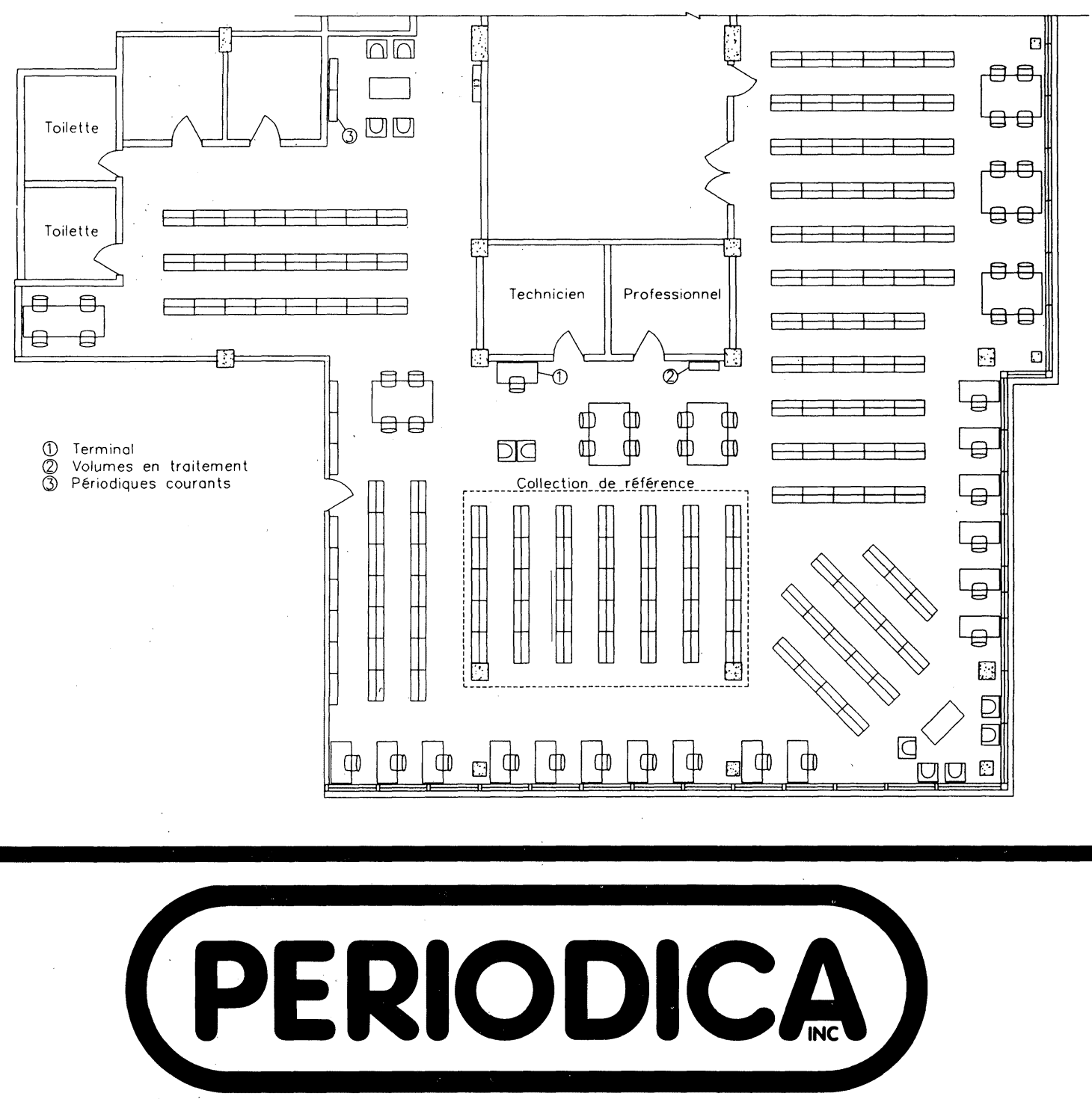

AGENCE INTERNATIONAL

INTERNATIONALE SUBSCRIPTION

D'ABONNEMENTS AGENCY

\section{Au service des collectivités et des bibliothèques depuis 1946}

- Les spécialistes de la gestion d'abonnements

- Un catalogue de 10,000 titres

- Des liens avec 2,500 éditeurs à travers le monde 


\section{Annexe 3 : La perception des usagers telle qu'illustrée par les sondages}

\section{SATISFACTION À L'ÉGARD DES SERVICES}

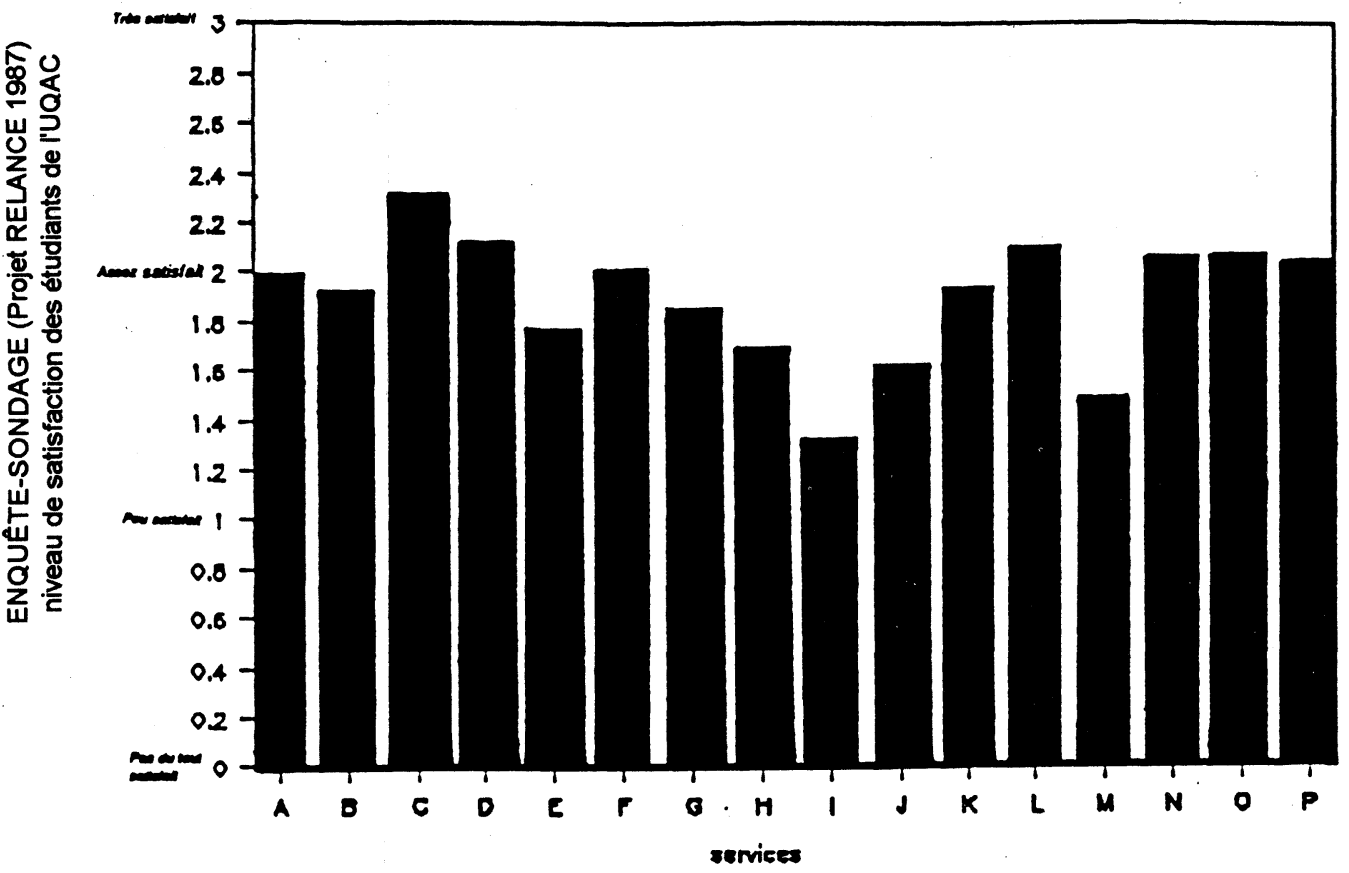

Le niveau de satisfaction de chaque élément a été fixé à partir de la moyenne des réponses obtenues.

La valeur du niveau de satisfaction s'évalue de la façon suivante :

TRÈS SATISFAIT :

ASSEZ SATISFAIT :

PEU SATISFAIT :

PAS SATISFAIT DU TOUT

a- salle de cours

b- laboratoires

c- bibliothèque

d- audiovisuel

e- informatique

f- vente et livraison de notes de cours

g- cafétéria

h- logement hors-campus

i- placement étudiant

$j$ - bourses et aide financière

k- pastorale

1- services de santé

$\mathrm{m}$ - orientation professionnelle

n- installations et équipements de sport

o- activités sportives

p- activités socio-culturelles

Pour le critère «Les outils de travail» (équipement audiovisuel, bibliothèque, etc...)

$\begin{array}{lll}\text { Rang } & \text { Université } & \text { Score } \\ 1 & \text { UQAC } & 1.24 \\ 2 & \text { Laval } & 0.94 \\ 3 & \text { Bishop } & 0.91 \\ 3 & \text { UQTR } & 0.91 \\ 4 & \text { UQAR } & 0.87 \\ 4 & \text { McGILL } & 0.87 \\ 5 & \text { UQAM } & 0.80 \\ 6 & \text { MONTREAL } & 0.72 \\ 7 & \text { SHERBROOKE } & 0.69 \\ 8 & \text { CONCORDIA } & 0.45 \\ 9 & \text { UQAH } & 0.10 \\ 10 & \text { UQAT } & 0.00\end{array}$

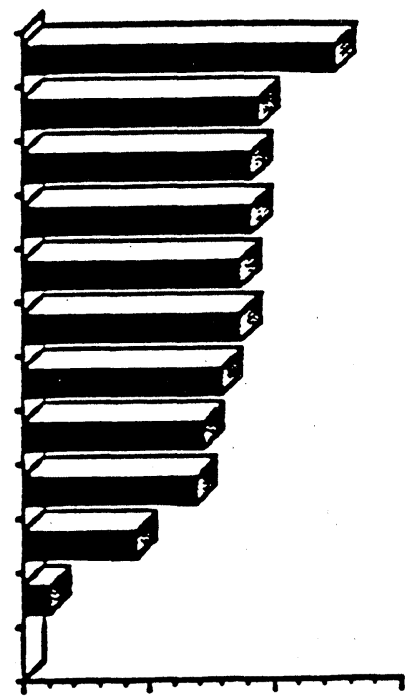

Moyenne $=0.78$

maximum possible $=2.00$

minimum possible $=-2.00$



